\title{
A PERCEPÇÃO DOS PROFISSIONAIS DE SAÚDE SOBRE A CULTURA DE SEGURANÇA DO PACIENTE EM UM HOSPITAL DE ENSINO DA GRANDE SÃO PAULO
}

\author{
Ana da Conceição de Lima Andrade ${ }^{1}$ \\ Gilmara Furtado dos Reis Penteado ${ }^{2}$ \\ Patricia do Prado Costa Braga ${ }^{3}$ \\ Sheila Pereira Mendes ${ }^{4}$ \\ Roseli Gomes Cavalini ${ }^{5}$
}

RESUMO: A perceção dos profissionais de saúde sobre a cultura de segurança do paciente em hospital de ensino da grande São Paulo. Trata-se de um projeto de intervenção cujo objetivo foi analisar a percepção do profissional da área de saúde, diante da cultura de segurança do paciente implantada. Aplicado na equipe multiprofissional de um hospital da grande São Paulo a partir do instrumento validado por REIS (2013) do qual se divide em duas partes: caracterização sociodemográfica e onze dimensões de cultura de segurança do paciente. As perguntas redigidas de forma afirmativas ou negativas obedecendo os critérios propostos na Escala Likert. A técnica empregada para facilitar a adesão e participação foi a disponibilidade computadores na entrada das unidades de internação e administração, bem como o envio de um link de preenchimento através da mídia social (WhatsApp). A amostra da pesquisa contou com a distribuição de IoIs questionários, como retorno de 772 (76\%), o que representa adequada adesão dos participantes. Os resultados da pesquisa foram divulgados via intranet da instituição para todos da equipe multiprofisional, dividido em duas etapas: pontos fortes e pontos frágeis. Ressalta-se que diante dos resultados apresentados foram ainda enviadas propostas com ações de reforço e melhoria. Eis alguns dos principais resultados: a) Staffing $19 \%$ acreditavam que a instituição apresenta quantidade, suficiente de colaboradores para lidar com a carga de trabalho, contudo, $36 \%$

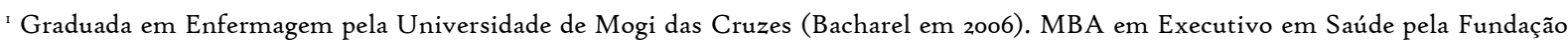
Getulio Vargas- FGV (2016) Especialização de Qualidade em Saúde e Segurança do Paciente, pela Escola Nacional de Saúde Pública Sergio Arouca - ENSP, da Fundação Oswaldo Cruz - FIOCRUZ ( 2020). Atualmente é Supervisora de Enfermagem da Associação Paulista para o Desenvolvimento da Medicina (SPDM ) Hospital das clínicas Luzia de Pinho Melo. E-mail: ana.andrade@hclpm.spdm.org.br.

${ }^{2}$ Graduada em Enfermagem pela Universidade de Mogi das Cruzes (Bacharel em 2006). MBA em Executivo em Saúde pela Fundação Getulio Vargas- FGV (2016) Especialização de Qualidade em Saúde e Segurança do Paciente, pela Escola Nacional de Saúde Pública Sergio Arouca - ENSP, da Fundação Oswaldo Cruz - FIOCRUZ ( 2020). Atualmente é Supervisora de Enfermagem da Associação Paulista para o Desenvolvimento da Medicina (SPDM ) Hospital das clínicas Luzia de Pinho. E-mail: Melo.gilmara.reis@hclpm.spdm.org.br.

${ }^{3}$ Graduada em Enfermagem pela Universidade de Mogi das Cruzes. Especialização de Qualidade em Saúde e Segurança do Paciente, pela Escola Nacional de Saúde Pública Sergio Arouca - ENSP, da Fundação Oswaldo Cruz - FIOCRUZ ( 2020). Atualmente é Gerente de Enfermagem da Associação Paulista para o Desenvolvimento da Medicina (SPDM) Hospital das clínicas Luzia de Pinho Melo.E-mail: patricia.braga@hclpm.spdm.org.br

${ }^{4}$ Graduada em Enfermagem pela Universidade Cruzeiro do Sul (Bacharel em 2005). Especialista em enfermagem em Unidade de Terapia Intensiva , pelo hospital Sírio Libanês (2007), Gestão em Enfermagem modalidade a distancia da Escola Paulista de Enfermagem - Pela Universidade Federal de São Paulo (2oir) e MBA em Saúde e em Controle de Infecção Hospitalar pela Faculdade Método de São Paulo (2015), Informatica em Saúde modalidade a distancia da Escola Paulista de Enfermagem, pela a Universidade Federal de São Paulo (2016).Especialização de Qualidade em Saúde e Segurança do Paciente, pela Escola Nacional de Saúde Pública Sergio Arouca - ENSP, da Fundação Oswaldo Cruz - FIOCRUZ ( 2020). Atualmente é Supervisora de Enfermagem da Associação Paulista para o Desenvolvimento da Medicina (SPDM ) Hospital das clínicas Luzia de Pinho Melo. E-mail:sheila.mendes@hclpm.spdm.org.br

${ }^{5}$ Graduação em Enfermagem pela União para a Formação, Educação e Cultura do ABC (20or). Espeicalização enfermagem em cuidados pacientes críticos pela FuABC. MBA Gestão em Saúde pela FGV. Atualmente é Diretora de enfermagem - Associação Paulista para o Desenvolvimento da Medicina (SPDM ) Hospital das clínicas Luzia de Pinho Melo. E-mail:roseli.gomes@hclpm.spdm.org.br.
} 
relataram que os colaboradores trabalham a mais do que o necessário, 50\% referiram que a instituição utiliza mais colaboradores temporários do que o necessário, mesmo com a intencionalidade de que seja prestado um melhor atendimento e $33 \%$ consideraram que trabalham em modo de crise, em resposta ao alto absenteísmo; b) Transferências internas e passagens de plantão - 32\% afirmaram perder informações durante as transferências internas do paciente, $31 \%$ apontaram as trocas de turno como problemáticas aos pacientes e $36 \%$ identificaram problemas frequentes ocorridos na troca de informação entre unidades; c) Respostas não punitivas aos erros - 31\% possuem a percepção de seus erros são levantados contra eles, $29 \%$ informaram que quando há evento relatado, o colaborar fica em evidência, sendo a pessoa avaliada e não o problema; $21 \%$ temem que erros cometidos sejam mantidos em seus arquivos pessoais. Considera-se que os resultados constituem-se oportunidades de melhoria e fortalecimento das ações de forma que a assistência à saúde seja com maior qualidade, minimizando os problemas existentes e futuramente ganhando maior maturidade na cultura de segurança do paciente.

Palavras-chave: Cultura de segurança do Paciente. Qualidade. Enfermagem. Segurança do paciente.

ABSTRACT: The perception of health professionals about the culture of patient safety in a teaching hospital in grande São Paulo. This is an intervention project whose objective was to analyze the perception of healthcare professionals, given the implanted patient safety culture. Applied in the multidisciplinary team of a hospital in greater São Paulo, using the instrument validated by REIS (2013), which is divided into two parts: sociodemographic characterization and eleven dimensions of patient safety culture. Questions written affirmatively or negatively following the criteria proposed in the Likert Scale. The technique used to facilitate adherence and participation was the availability of computers at the entrance to the inpatient and administration units, as well as sending a link to fill in through social media (WhatsApp). The research sample included the distribution of rors questionnaires, with a return of 772 ( $76 \%)$, which represents adequate participation by the participants. The survey results were disseminated via the institution's intranet to all of the multidisciplinary team, divided into two stages: strengths and weaknesses. It is noteworthy that in view of the results presented, proposals were also sent with reinforcement and improvement actions. Here are some of the main results: a) Staffing $19 \%$ believed that the institution had a sufficient amount of employees to handle the workload, however, $36 \%$ reported that employees work more than necessary, 50\% reported that the institution uses more temporary employees than necessary, even with the intention of providing a better attendance and $33 \%$ considered they work in crisis mode, in response to high absenteeism; b) Internal transfers and shift changes - 32\% said they lost information during internal patient transfers, $31 \%$ pointed out shift changes as problematic for patients and $36 \%$ identified frequent problems in the exchange of information between units; c) Nonpunitive responses to errors - 31\% have the perception that their errors are raised against them, $29 \%$ reported that when there is an event reported, collaborating is in evidence, with the person being evaluated and not the problem; 21\% fear that mistakes made will be kept in their personal files. It is considered that the results are opportunities to improve and strengthen actions so that health care is of greater quality, minimizing existing problems and, in the future, gaining greater maturity in the culture of patient safety.

Keywords: Patient safety culture. Quality. Nursing. Patient safety. 


\section{INTRODUÇÃO}

A segurança do paciente é definida como a diminuição do risco de danos desnecessários associados à atenção à saúde, até o mínimo aceitável. O ambiente hospitalar proporciona aos assistidos procedimentos e tratamentos complexos, o que potencializa a chance de ocorrer danos (Rede Brasileira de Enfermagem e Segurança do Paciente, 2013). Esta temática relaciona-se intrinsicamente com a qualidade dos serviços prestados e vem sendo discutida pelos gestores de prestadores de serviços de saúde, entidades de classe e órgãos governamentais (TASE, 2013).

A cultura da segurança é definida pela Agência Nacional de Vigilância Sanitária, na $\mathrm{RDC} \mathrm{n}^{\mathrm{o}}$ 36/2013 como “conjunto de valores, atitudes, competências e comportamentos que determinam o comprometimento com a gestão da saúde e da segurança, substituindo a culpa e a punição pela oportunidade de aprender com as falhas e melhorar a atenção à saúde” (BRASIL, 2013). Dentre as características de uma cultura sólida de segurança algumas ações merecem destaque como o reconhecimento da evitabilidade do erro, a discussão e aprendizado a partir dos erros, a identificação proativa das ameaças, e a inclusão de um sistema não punitivo para o relato e análise de Eventos Adversos são ações que merecem destaque (PADILHA, 200I; BOGNÁR, 2008).

Podemos verificar que a cultura de segurança do paciente pode conter a seguinte definição: modelo integrado de comportamento individual e organizacional, de acordo com crenças e valores comuns para assim minimizar os danos ao paciente que podem resultar dos processos de prestação de cuidados (Kristensen, Mainz, Bartels, 2007).

Em 20ı, Sorra e Dyer ampliam o conceito de cultura de segurança do paciente como algo que é visto, crescentemente, como ponto central para o entendimento de segurança do paciente nas instituições que provem cuidados à saúde. A cultura de segurança do paciente refere-se, portanto, ao gerenciamento e valores da equipe, bem como, os sentimentos e normas sobre o que é importante na organização de cuidados a saúde, acrescido, de como os membros da equipe devem se comportar, onde inclui-se as atitudes e ações apropriadas além dos processos e procedimentos que devem ser revistos com relação à segurança do paciente.

Permanece a tendência de promover o envolvimento de todos os níveis da organização, desde o gerencial até os profissionais que atuam na linha de frente, estimulando o reconhecimento das circunstâncias de risco na organização (GALT KA, 2010). A enfermagem desempenha um papel fundamental nesse reconhecimento, tanto pelo seu 
contingente como pela sua proximidade constante e ininterrupta na assistência ao paciente, quando opta a identificar estes riscos, contribui na oferta de valiosas sugestões de melhoria. Nesse sentido, tem sido realizados estudos com os profissionais, para que sejam avaliados os aspectos existentes na cultura do seu ambiente de trabalho visto os mesmos serem considerados e utilizados no fortalecimento da segurança do paciente (NIEVA, 2003; PANOZZO, 2010).

Contudo, para se promover uma cultura que vise à segurança do paciente como prioridade, primeiramente é fundamental avaliar como a cultura de segurança do paciente presente nas organizações de saúde, tem sido construída e vivida, consequentemente, planejar intervenções específicas em busca de melhores resultados (SORRA, 2010).

Para que haja melhoria da qualidade e aumento da segurança do paciente é necessário, o trabalho integrado das equipes interdisciplinares com o apoio da alta direção, ou seja, apoio de pessoas que tomam as decisões sobre o destino de uma organização (DONALDSON, 1999) e cujos esforços despendidos para a correção de processos inseguros, alinhados à visão, missão e valores da organização, tornam possível uma mudança na cultura de segurança do paciente e na cultura organizacional, refletindo nos comportamentos compartilhados, crenças, atitudes e valores a respeito de metas, funções e procedimentos (SCHEIN, I996; BRASIL 2013).

Discorrer sobre a cultura da segurança do paciente é de suma importância para avaliar como o tema vem sendo tratado atualmente, visto que suas práticas bem estruturadas nas instituições hospitalares incorporam ações, com o objetivo de oferecer assistência de excelência, assegurar a satisfação dos usuários dos serviços, reduzir custos, e acima de tudo reduzir os riscos inerentes à prestação de cuidados em saúde, um estudo publicado em 2.013 estimou que mortes associadas a danos evitáveis acometam mais de 400.000 pessoas por ano e cerca de 4 a 8 milhões de pessoas são acometidos por danos graves, o que significa que diariamente ocorram, em média, I.096 mortes e 10.949 a 20.918 danos graves, por estes motivos o presente trabalho visa colaborar com a sociedade e com os profissionais de saúde na busca pela prestação de serviços em saúde mais segura e livre de danos evitáveis, além de estimular a curiosidade e o olhar da sociedade acadêmica para este assunto tão delicado de ser analisado e tão essencial para ser discutido.

O estudo realizado, a experiência em atividades assistenciais e de gestão do cuidado no contexto hospitalar e a importância da consolidação da cultura de segurança para efetiva 
prestação de cuidados em saúde de excelência, inspiraram a explorar a percepção do profissional da área de saúde, no hospital de ensino da grande São Paulo, diante da cultura de segurança do paciente implantada.

A busca por esta resposta inquietante será desafiadora, pois trará uma visão norteadora das fortalezas e fraquezas institucional, permitindo atuar, posteriormente, diretamente nos pontos a serem melhorados, apoiados nos pontos fortes, visto a cultura de segurança do paciente a ser considerado um importante componente estrutural dos serviços que favorece a implantação de práticas seguras e diminuição de incidentes de segurança. Sendo seu objetivo geral, analisar a percepção do profissional da área de saúde diante da cultura de segurança do paciente implantada e específico, identificar quais as dimensões precisam ser desenvolvidas para melhorar a efetividade da cultura de segurança do paciente dentro da instituição de saúde.

\section{REFERENCIAL TEÓRICO}

\subsection{Cultura}

Impossível referenciarmos sobre cultura de segurança sem antes mencionarmos o

conceito da palavra cultura. Para HELMAN (2009, p.12) trata-se de um conjunto de orientações (tanto explícitas como implícitas) que os indivíduos herdam como membros de uma sociedade particular, as quais lhe dizem como ver o mundo, como experimentá-lo emocionalmente e comportar-se em relação às outras pessoas, às forças sobrenaturais ou aos Deuses ou ao ambiente natural. A autora acrescenta que a cultura oferece ao indivíduo uma maneira de transmitir essas orientações às próximas gerações.

No cuidado clínico, o mau uso do conceito de cultura ocorre quando sua influência é enfantizada de maneira excessiva, na interpretação de como algumas pessoas apresentam seus sintomas aos profissionais de saúde. De um modo geral, a cultura não pode ser considerada uma lacuna, pois a cultura pura dificilmente existe, sendo ela, apenas, a peça de uma mistura complexa e diversificada de fatores que influenciam as crenças das pessoas, sobre a forma como elas vivem em seu cotidiano e sobre a sua saúde e doença.

Já no dicionário online cultura é o conjunto dos hábitos sociais e religiosos, das manifestações intelectuais e artísticas, que caracteriza uma sociedade; são normas de comportamento, saberes, hábitos ou crenças que diferenciam um grupo de outro: provêm de culturas distintas. Conjunto dos conhecimentos adquiridos; instrução: sujeito sem cultura. 
Expressão ou estágio evolutivo das tradições e valores de uma região, num período determinado: cultura católica. Aplicação do espírito a uma coisa: a cultura das ciências.

Cultura originariamente significa lavoura, cultivo agrícola, ela também sugere tanto regulação como crescimento espontâneo. O cultural é o que podemos mudar, mas o material a ser alterado tem sua própria existência independente, a qual então lhe empresta algo da perseverança da natureza. Mas cultura também é uma questão de seguir regras e isso também envolve uma interação entre o regulado e o não regulado (EAGLETO, 2003).

Cultura considerado como algo muito profundo que une grupos e determina regras, possuindo forte influência sobre as pessoas sendo extremamente difícil e trabalhosa sua modificação, daí necessitar de tempo e engajamento para transformações culturais, além de se fazer acreditar por todos os envolvidos.

\subsection{Cultura Organizacional}

De acordo com Freitas (199I) Cultura Organizacional é o modelo dos pressupostos básicos, que um dado grupo inventou, descobriu ou desenvolveu no processo de aprendizagem, para lidar com os problemas de adaptação externa e integração interna. Uma vez que os pressupostos tenham funcionado bem o suficiente para serem considerados válidos, eles são ensinados aos demais membros da organização como a maneira certa de se perceber, pensar e sentir em relação aqueles problemas. A descrição dos elementos que constituem a cultura organizacional, a forma como eles funcionam e, ainda, as mudanças que eles provocam no comportamento são maneiras de dar ao assunto um tratamento mais concreto e de mais fácil identificação. Nesse sentido, é bastante comum encontrarmos a cultura sendo conceituada a partir dos seus próprios elementos institucionais como missão, visão, valores e política.

A cultura organizacional pode ser concebida como um conjunto de pressupostos básicos que um grupo inventou, descobriu ou desenvolveu ao aprender como lidar com os problemas de adaptação externa e integração interna e que funcionou bem o suficiente para ser considerado válido e ensinado a novos membros como a forma correta de perceber, pensar e sentir, em relação a esses problemas (Schein, 1986; Fleury, 1996).

Saraiva (2002) afirma que a cultura organizacional tem sido colocada por diversos estudiosos como importante aspecto da análise organizacional, capaz de oferecer parâmetros úteis para uma compreensão ampliada do comportamento da organização. A construção de 
análises baseadas em tal perspectiva possibilita uma visão diversa do que normalmente tem sido feito na administração, pois considera a influência de elementos não objetivos, que têm sua influência ofuscada pelo pragmatismo predominante na literatura da área.

O conceito de cultura é necessário e muito utilizado porque atende a várias necessidades e vários interesses da sociedade e dos próprios pesquisadores. A cultura implica continuação, denota demonstrações conceituais, serve como fator aglutinador para levar os membros do grupo em direção ao consenso, implica dinâmica e padronização. Falar em cultura implica falar sobre a capacidade de adaptação do indivíduo à realidade do grupo no qual está inserido. As organizações são instrumentos criados para atingirem outros fins, a organização depende das pessoas para atingir seus objetivos, é por meio da interação entre as pessoas que se definem os propósitos das organizações. É por isso que as idéias sobre tarefas, metas, propósitos e objetivos se tornaram conceitos organizacionais tão fundamentais. (Pires e Macedo, 2006).

O clima e a cultura organizacional oferecem perspectivas sobrepostas para a compreensão de experiências que as pessoas vivenciam em ambientes organizacionais (SCHNEIDER, 2013). Apesar de constantemente utilizadas como sinônimos, cultura organizacional e clima organizacional possuem conceitos diferentes (COLLA, 2005).

Fleury (1996) afirma que conhecer sobre a cultura organizacional é uma categoria metodológica importante para a compreensão de aspectos formadores da identidade institucional. Dessa forma, essa autora desenvolveu um modelo de observação que propõe que o estudo de cultura organizacional foque nos seguintes aspectos:

Histórico da organização: o momento de criação, inserção no contexto político e econômico da época e os seus fundadores; Processo de socialização dos novos membros: programas de treinamento e integração e outros processos que visem à reprodução do universo simbólico da organização;

Políticas de recursos humanos: análise de políticas explícitas e implícitas de recursos humanos de uma organização, observação de consistências e inconsistências;

Processo de comunicação: criação, transmissão e cristalização do universo simbólico, meios formais orais e escritos, e meios informais; 
Organização do processo de trabalho: identificação das categorias presentes na relação detrabalho.

Fleury (1996) cita que para criar e manter a cultura, normas e valores devem ser afirmados e comunicados aos membros da organização de uma forma tangível. A parte tangível constituída por símbolos, ritos, rituais, mitos, estórias, gestos e artefatos. Salienta que, o mapeamento dos sistemas de comunicação, que inclui os meios, instrumentos, veículos, como a relação entre quem se comunica é fundamental para apreensão desse universo simbólico.

Como podemos notar a cultura organizacional é fator determinante para o sucesso dos empreendimentos de uma instituição, pois se trata dos valores individuais e do grupo além da sua percepção de mundo e modo de agir; ela é um facilitador na retenção de talentos e no agrupamento de profissionais com o mesmo ideal, a cultura organizacional denota bem o perfil da instituição e suas ferramentas de atuação.

\subsection{Segurança do Paciente}

A Organização Mundial da Saúde (OMS) define segurança do paciente como a redução do risco de danos desnecessários a um mínimo aceitável, considerado componente constante e intimamente relacionado com o atendimento ao paciente (WHO, 2009).

A segurança do paciente é influenciada, apesar dos avanços na área de saúde, pelas iatrogenias cometidas pelos profissionais, as quais refletem diretamente na qualidade de vida dos clientes, provocando consequências desagradáveis tanto para os pacientes como para os profissionais e para a organização hospitalar (MIASSO et al., 2006).

A segurança do paciente é definida como o ato de evitar, prevenir ou melhorar os resultados adversos ou lesões originadas no processo de atendimento médico-hospitalar e domiciliar (RIGOBELLO, 2012). O tema é um grave problema de saúde pública global. Dados apontam que, em países desenvolvidos, um em cada io pacientes é prejudicado ao receber cuidados hospitalares. O risco de infecção associado aos cuidados de saúde em alguns países em desenvolvimento é de até 20 vezes maior que em países desenvolvidos (BARROS, 2013).

Nos cuidados da segurança em saúde o médico Ignaz Phillip Semmelweis (I818-1865) contribuiu para os avanços na assistência obstétrica, em I846 tornou-se médico assistente da maternidade do Hospital de Viena, local este que apresentava altos índices de mortes de 
parturientes e neonatos recorrentes de infecções puerperais, através de suas pesquisas identificou que as práticas realizadas por estudantes de medicina que circulavam entre a enfermaria e a sala de autópsia era o motivo de tal agravo, evidenciando a febre puerperal pela contaminação cruzada, através dos resultados de seus estudos impôs a higienização das mãos antes da realização de qualquer procedimento (SILVA; MATTOS, 2015). Além dos médicos citados, a pioneira em enfermagem, Florence Nightingale (1820-1910) declarou na conclusão de uma de suas obras em 1859: "Pode parecer talvez um estranho princípio, enunciar como primeiro dever de um hospital não causar mal ao paciente" (PEDREIRA, p.I). Florence transfigurou a enfermagem criando maneiras de prestar assistência visando a segurança, utilizando processos sistematizados para monitorar a incidência de infecções e a recuperação dos pacientes, suas idéias inovadoras se fazem presentes após tantas gerações nos cuidados em saúde, suas pesquisas e teorias fundamentam a prática da enfermagem (PEDREIRA, 2009).

Para o The Canadian Patient Safety Dictionary (2003) a segurança do paciente é a redução e mitigação de atos não seguros dentro do sistema de assistência à saúde, assim como a utilização de boas práticas para alcançar resultados ótimos para o paciente.

No Brasil, a preocupação com a segurança do paciente começou a surgir em meados dos anos 90, quando as instituições públicas e privadas passam a adotar medidas para melhoria da qualidade hospitalar. Em i99i foi criado o Programa de Controle da Qualidade Hospitalar (CQH) (D“e INNOCENZO et al.,2006).

De acordo com OLIVEIRA et. al. (2014), há dez anos, foi divulgado o relatório: (Errar é humano: construindo um sistema de saúde mais seguro) pelo Instituto de Medicina dos Estados Unidos, onde foi descrito através de pesquisas realizadas por meio de análises de prontuários a ocorrência de I8o.ooo óbitos por ano naquele país. Após a publicação deste relatório, a Organização Mundial de Saúde (OMS) lançou a Aliança Mundial para a Segurança do Paciente em 2004, em prol do compromisso no desenvolvimento de políticas públicas e práticas direcionadas para a segurança do paciente, o Brasil firmou esse compromisso, embora o sistema de saúde brasileiro aponte aspectos relevantes e positivos como o sistema nacional de transplantes e a cobertura universal de vacinação, é motivo de apreensão o alto índice de eventos adversos relativos a infecções hospitalares e medicações, sendo relacionados com a falta de políticas que incentivem as instituições a aderirem programas de acreditação e qualidade (CAPUCHO; CASSIANI, 2013). 
Vale ressaltar que a falta de conhecimento e segurança dos profissionais de saúde, fazem com que acabem realizando procedimentos errados, colocando em risco a vida do paciente. Por esse motivo garantir um cuidado seguro tem sido um desafio constante para as organizações de saúde (TAVARES, 2013; FREITAS et al., 2014).

Raduenz et al (2010), defende que os profissionais são os responsáveis pelo planejamento e intervenção apropriada com a finalidade de manter o ambiente seguro, sendo portanto, vital o desenvolvimento de pesquisa em enfermagem sobre segurança do paciente.

Segundo Pedreira (2009), os profissionais de enfermagem são responsáveis por grande parte das ações assistenciais e, portanto, encontram-se em posição privilegiada para reduzir a possibilidade de incidentes que atingem o paciente, além de detectar as complicações precocemente e realizar as condutas necessárias para minimizarem os danos.

Sendo assim, podemos ratificar que a segurança do paciente é a base fundamental para assegurarmos o cuidado à saúde, desta forma se faz necessário práticas e hábitos seguros, reduzindo danos a fim de promover uma assistência segura aos pacientes.

\subsection{Cultura de Segurança do Paciente}

Para Claro (20II), as instituições hospitalares têm incorporado ações em prol da cultura do paciente, com o objetivo de oferecer assistência de excelência, diminuir custos e assegurar satisfação. Quando a segurança é instituída como processo cultural, constata-se maior consciência profissional quanto à cultura de segurança do paciente e ao compromisso ético do gerenciamento de risco para o profissional e para o paciente.

A segurança no cuidado ao paciente tem sido cada vez mais evidenciada em estudos pelos profissionais da saúde ao longo dos anos, especialmente nas duas últimas décadas. A enfermagem tem na sua origem a preocupação em promover a segurança do paciente. Um dos marcos da enfermagem, Florence Nightingale, sustentava o princípio que o primeiro dever de um hospital deveria ser o de não causar dano ao paciente. Nos seus estudos iniciais sobre epidemiologia, constatava que o índice de mortalidade era maior entre os pacientes tratados em hospitais, do que entre os pacientes tratados em casa (NIGHTINGALE, I863).

Para Batalha e Melleiro (2015), a consolidação da segurança do paciente se configura como subsídio para as propostas de melhoria da qualidade, pois as suas construções permitem remodelar os processos de trabalho, fazendo com que estratégias seguras aprimorem a assistência em saúde com a redução, a um mínimo aceitável, do risco de dano 
desnecessário associado ao cuidado de saúde. A cultura de segurança de uma organização é o produto dos valores individuais e do grupo, atitudes, percepções, competências e padrões de comportamento que determinam o compromisso, com e o estilo e proficiência de gestão da saúde e segurança de uma organização. Organizações com cultura de segurança positiva são aquelas caracterizadas por comunicação baseada na confiança mútua, por percepções compartilhadas da importância da segurança e na confiança na eficácia de medidas preventivas caracterizada pela gestão de segurança.

O desenvolvimento da cultura de segurança nas instituições foi uma das recomendações feitas pelo IOM para auxiliar os hospitais a melhorar a segurança do paciente. Nos últimos anos foram produzidas publicações sobre essa temática, principalmente nos países desenvolvidos. A cultura de uma organização de saúde tem sido identificada, como um fator crítico para a segurança do paciente e um componente fundamental para o desenvolvimento de estratégias, ao propiciar implementações bem sucedidas de iniciativas de melhoria da qualidade (SPEROFF, 2010).

Tomazone et al (2014) considera que a cultura de segurança do paciente está associada tanto aos fatores individuais como coletivos, ou seja, o modo de pensar, agir e fazer a segurança no local de trabalho. Assim, a formação dessa perspectiva da cultura é construída coletivamente com o passar do tempo e das experiências no ambiente de trabalho, onde cada um contribui com seus valores e compartilha a prioridade da segurança do paciente. Dessa maneira, a cultura de segurança também pode ser influenciada pelo cargo que o profissional ocupa e pelo seu tempo de trabalho na profissão, assim como pelo tempo de trabalho em determinada instituição ou unidade, passando a tomar suas decisões de acordo com o clima de segurança predominante em cada local.

Nem todas as dimensões da cultura de segurança do paciente têm o mesmo peso quando se considera a cultura organizacional. Assim, é relevante que se analise a cultura de segurança nas organizações de saúde, com o intuito de identificar as dimensões que necessitam ser introduzidas ou aprimoradas. Além disso, serve como direcionamento para o gestor na implantação de estratégias, que fortaleçam e incrementem a questão de segurança nas organizações, principalmente tendo em vista que mudança das percepções sobre a cultura de segurança pode consumir recursos e esforços consideráveis. (SILVA et al, 2016).

Atualmente, as Instituições de saúde apresentam um crescente interesse em pesquisas relacionadas à avaliação da cultura de segurança do paciente, sendo considerada a primeira 
etapa na construção da comissão de segurança do paciente. Neste sentido, mensurar o clima de segurança por meio da percepção dos profissionais deve ser um ponto de partida para as instituições, por trata-se de um componente mensurável da cultura de segurança do paciente que pode ser compreendida como a redução, a um mínimo aceitável, do risco de dano desnecessário associado ao cuidado de saúde.

Para que haja uma cultura de segurança do paciente positiva, as organizações de saúde devem priorizar a comunicação baseada na confiança entre os profissionais, com consciência por parte de todos da importância dessa confiança, valorização das medidas preventivas que devam ser implementadas a fim de melhorar a qualidade do cuidado prestado e não ocorrência de punição frente aos erros, mas somente das condutas inapropriadas quando existirem. $\mathrm{O}$ ambiente hospitalar deve ser seguro para que os profissionais possam relatar os erros ocorridos, conversar sobre eles, analisa-los junto às situações que os precederam, identificar os pontos frágeis dos processos a fim de repará-los e discutir estratégias de melhorias (REIS et al.,2013).

A necessidade do fortalecimento de uma cultura de segurança no nível organizacional é apontada como medida fundamental ao processo de melhoria da segurança do paciente no contexto hospitalar (KOHN, 200o). A cultura de segurança é definida como o produto de valores, atitudes, competências e padrões de comportamento individuais e de grupo, os quais determinam o compromisso, o estilo e a proficiência da administração de uma organização saudável e segura. (HEALTH AND SAFETY COMISSION, 1993).

Reason (2004) enfatiza a necessidade de uma cultura de segurança justa nas organizações de saúde, onde não haja punição para os erros, mas sim para condutas impróprias; que seja uma cultura voltada para o relato dos erros em um ambiente seguro, que estimule as pessoas a conversarem sobre as falhas ocorridas, analisar as situações as quais as precederam, identificando os pontos frágeis do sistema para que esses sejam reparados.

De acordo com o Ministério da Saúde (2013) a instituição do Programa Nacional de Segurança do Paciente (PNSP) se deu a fim de conceder um retorno ao apelo individual e coletivo dos profissionais da saúde e da população em geral, por uma atenção segura, livre de incidentes que possam ocasionar danos à saúde do indivíduo.

Fica evidente que a cultura de segurança do paciente é algo construído por meio da comunicação efetiva, da constituição de uma relação de confiança entre os gestores e as 
equipes de atuação com a tratativa dos eventos de maneira educativa e com foco no processo. Somado a isso, a cultura de segurança é uma excelente ferramenta de avaliação da qualidade da gestão dos administradores atuantes e da qualidade do serviço de saúde prestado, além da segurança fornecida ao usuário e aos prestadores de serviço.

\subsection{Dimensões de Cultura de Segurança do Paciente}

São onze as dimensões de Cultura de Segurança do Paciente, que foram mensuradas na pesquisa realizada no hospital de ensino da grande São Paulo e serão dissertadas a seguir.

\section{Dimensão Cultura de Segurança do Paciente: “Abertura de Comunicação"}

Conforme afirma Peruzzo (2002), a comunicação é um mecanismo facilitador da ampliação da cidadania, pois permite à pessoa se tornar sujeito de atividades de ação comunitária, que resulta num processo educativo. Desse modo, a comunicação deixa de ser vista como instrumento e passa a desempenhar um papel transformador e educativo, favorecendo o diálogo permanente entre os diferentes atores sociais, oportunizando a integração e a participação dos públicos no processo de construção da cidadania.

A comunicação eficaz entre os profissionais é crucial para o atendimento seguro, especialmente em serviços complexos e que envolvem múltiplos profissionais (Clark, 2018) como, por exemplo, ambientes de internação cirúrgica. Neste sentido, a Organização Mundial de Saúde entre 2007-2008 lançou o segundo desafio global (Cirurgias Seguras Salvam Vidas), no qual enfatizou a importância de promover a comunicação entre os membros da equipe cirúrgica por meio da instrumentalização da lista de verificação de segurança cirúrgica em formato de check list para evitar falhas operatórias e assegurar o cuidado seguro $(\mathrm{CH}, 2009)$.

$\mathrm{O}$ ato da comunicação é fundamental para o desenvolvimento do trabalho dos enfermeiros junto à equipe, para a transmissão de uma informação universal, além de exercer influência direta sobre os indivíduos. A comunicação é uma habilidade humana que torna possível a manifestação e exteriorização do que se passa interiormente (Silva, 2007).

\section{I Dimensão Cultura de Segurança do Paciente: "Frequência de eventos relatados"}

Nas organizações de saúde, uma cultura de segurança resulta de valores individuais e de grupo, atitudes, percepções, competências, e padrões de comportamento que 
determinam o compromisso, o estilo e a competência da gestão da segurança e saúde da organização (COMISSION,1993).

De acordo com Thomas e Brennan (2000), um EA pode ser definido como um dano causado durante a prestação de cuidados de saúde ou que resulta dessa prestação, o que pode conduzir a uma de duas situações: ou uma estadia prolongada e não esperada na instituição de saúde, ou incapacidade mantida após a alta.

Outros autores definiram EA como um evento indesejável que, em condições normais, não surge como consequência da história natural da doença, nem dos efeitos previstos da terapêutica instituída (Australia, 200I).

Para Reason 2000, o conceito atual de segurança do paciente aponta como principais fatores responsáveis pela ocorrência de EA, as deficiências do sistema de prestação de cuidados de saúde, em sua concepção, organização e funcionamento, em vez de responsabilizar os profissionais ou produtos isoladamente. A premissa é de que os seres humanos cometem falhas, e que, portanto, erros são esperados. Os erros são consequências, não causas. E embora não se possa mudar a condição humana, é possível atuar naquelas sobre as quais os seres humanos trabalham, criando defesas no sistema

As organizações devem adotar uma abordagem de aprendizagem com o erro, examinando os eventos que conduzem ao incidente, propondo mudanças razoáveis no sistema que permitam a prevenção dos EA, e implementando essas mudanças de forma efetiva. Esta abordagem terá como objetivo reduzir a probabilidade de ocorrência de incidentes, uma vez que a segurança depende da criação de sistemas de antecipação e/ou prevenção dos EA (Carneiro, 2010).

Os investigadores e profissionais empenhados na melhoria da qualidade dos cuidados de saúde têm reunido esforços para avaliar, reduzir e monitorizar a ocorrência de EA nos hospitais. No entanto, a questão que se mantém é se os métodos que se têm utilizado para avaliar a frequência dos EA são os mais adequados em termos de eficiência, efetividade e eficácia (Thomas; Petersen, 2003).

\subsection{Dimensão Cultura de Segurança do Paciente: "Feedback e Comunicação de eventos"}

Um dos desafios para garantir a segurança do paciente no ambiente hospitalar é enfatizar a comunicação efetiva, como meta a ser atingida pela equipe interdisciplinar, como também, proporcionar um ambiente de trabalho harmonioso com assistência livre de danos. 
Nesse sentido, a comunicação é fundamental para um bom desenvolvimento do trabalho, pois é o elo de interação que fortalece o vínculo entre a equipe interdisciplinar e o cliente (Nogueira; Rodrigues, 2015).

Estudos mostram que a comunicação e o trabalho em equipe na saúde são determinantes na qualidade da assistência ao paciente. Segundo a Organização Mundial de Saúde (OMS), um em cada dez pacientes no mundo é vítima de erros e eventos adversos relacionados à assistência aos pacientes, que a levaram a investigar e propor soluções para prevenção dos danos. No Brasil, a importância da comunicação efetiva como meta de segurança do paciente foi difundida após publicação de Portaria Ministerial 529/2013 (Brasil, 2013; Marques; Lieber, 2014).

Campos e Melo (2002), reportam-se diretamente ao enfermeiro como agente potencialmente integrador no processo de comunicação na organização, revelando que se espera que o enfermeiro seja o elo na cadeia de comunicação, por estar em contato com toda equipe multiprofissional sendo a ele que a administração do hospital se dirige para que as normas e protocolos sejam implantados, quer para assuntos ligados diretamente a enfermagem ou não.

\subsection{Dimensão Cultura de Segurança do Paciente: “Apoio da gestão hospitalar para segurança do paciente"}

A cultura de segurança do paciente diz respeito ao gerenciamento e valores da equipe, igualmente aos sentimentos e normas sobre o que é importante na organização de saúde; como os membros da equipe devem comportar-se; quais as atitudes e ações são apropriadas ou inapropriadas; e quais os processos e procedimentos devem ser recompensados ou punidos com relação à segurança do paciente (Sorra, 2010).

Os modelos de gestão vigentes nas organizações de saúde, muitas vezes, apresentam estruturas verticais, centralizadas e hierárquicas. Nestes modelos há maior amplitude de supervisão dos trabalhadores, maior controle de ação e anulação de decisões do trabalhador sobre o trabalho. Entretanto, este modelo nem sempre atende as necessidades dos gerentes, trabalhadores e usuários, sendo necessária a substituição desses modelos, por vezes ultrapassados, por modelos inovadores, flexíveis, horizontalizados e que motivem mais as pessoas no seu ambiente de trabalho (Bernades, 20II)

Geralmente quando ocorrem erros ou EA, a resposta humana é encontrar culpados e 
solicitar compensações e retratações, procurando assegurar-se de que a falha não ocorrerá novamente, nessa compreensão percebe-se a necessidade de se modificar a cultura organizacional e torná-la mais voltada para a segurança do paciente de modo a dar resultados satisfatórios e efetivos e não apenas agir após o acontecimento de um EA (Pedreira, 2009).

\subsection{Cultura de Segurança do Paciente: "Trabalho em equipe no âmbito das unidades"}

Como afirma Wachter (2010), as equipes que funcionam adequadamente determinam uma série de estratégias, enfatizando, principalmente, programas de treinamento em gestão de recursos humanos, para melhorar a comunicação e o trabalho em equipe. As primeiras estratégias concentram-se em formas de diminuir os níveis de autoridades, esses esforços podem incluir técnicas simples, como fazer o líder apresentar-se, saber o nome dos outros profissionais, admitir as suas limitações e aceitar de forma natural informações e alertas de todos os membros que compõe a equipe.

O trabalho multiprofissional numa perspectiva transdisciplinar requer humildade e disponibilidade por parte de cada profissional, pois é, em suma, um movimento de reconhecimento de posições diferentes em relação a um mesmo objeto, e gerar novos dispositivos é a segunda meta para se iniciar um trabalho transdisciplinar em que os profissionais possam se ajudar reciprocamente em suas dificuldades (Caon 1998).

\subsection{Dimensão cultura de segurança do paciente: "Transferências internas e passagens de plantão"}

A passagem de plantão, entrega de turno ou troca de turno é uma atividade que proporciona a identificação de problemas singulares a cada paciente, a definição de necessidades para o planejamento e a execução de medidas de enfermagem que possibilitem a eficácia do tratamento. Portanto, a comunicação e as informações sobre os pacientes são essenciais entre os profissionais de saúde como forma de garantir a continuidade dos cuidados; a passagem de plantão é considerada um elo entre as equipe e um momento oportuno para realizar um feedback sobre o trabalho desenvolvido (Zoehler, Lima, 200o; Silva, Campos, 2007; Portal, Magalhães,2008.

\subsection{Dimensão cultura de segurança do paciente: "Aprendizado e melhoria contínua"}

A questão educativa e de aprendizado constante é fator primordial para a melhoria da 
qualidade na assistência prestada pelos serviços de saúde. A ênfase deve ser dada para as políticas de recursos humanos, que devem ser pautadas na educação continuada e permanente dos profissionais de saúde que ali desempenham a sua função (Paschoal, Mantovani, Lacerda, 2006; Mira et al., 2011; Batista, 2012)

A segurança do paciente vem sendo um assunto discutido cada vez mais na atualidade, como uma das principais maneiras de assegurar a qualidade do cuidado ao paciente/cliente durante sua hospitalização, ou seja, não prolongar seu tempo de internação, consequentemente gerando menos gastos hospitalares e, assim, proporcionando um atendimento de qualidade (Reis, 2013).

3.7 Dimensão cultura de segurança do paciente: "Expectativa da promoção da segurança pelos supervisores e gerentes"

De acordo com Massarolo e Fernandes (2010), gerenciar em enfermagem pressupõe tomada de decisões, que dependem do grau de autonomia do gerente de enfermagem e de como se dá a sua relação com as pessoas e com a própria política da instituição.

A avaliação da cultura de segurança do paciente nas organizações de saúde tem um

papel fundamental na promoção do cuidado seguro, tendo em vista que esses estudos apontam as áreas que necessitam de melhorias e, assim, ajudam a nortear ações e atitudes, visando ao melhor desempenho global (Jardali,2014).

\subsection{Dimensão cultura de segurança do paciente: “ Percepção geral sobre segurança"}

O relatório To err is human que divulgou dados alarmantes sobre EAs (Eventos Adversos), o documento de referência para a segurança do paciente da ANVISA, fala que a notícia ganhou notoriedade e, diante do cenário apresentado, governos e organizações internacionais se mobilizaram. Assim, iniciaram-se trabalhos para apoiar as estratégias nacionais e internacionais para a prevenção e a mitigação de falhas no cuidado à saúde (BRASIL, 2014).Nessa perspectiva, a segurança do paciente torna-se foco de pesquisadores de todo o mundo assumindo uma dimensão fundamental para qualidade em saúde. A Organização Mundial de Saúde (OMS) estabeleceu, em 2004, a Aliança Mundial para a Segurança do Paciente (World Alliance for Patient Safety) ao reconhecer globalmente a magnitude do fenômeno (Reis, 2013).

Em 2010, o COREN/SP e REBRAENSP/SP lançam a cartilha "io Passos para a 
Segurança do Paciente" e a mesma foi elaborada com o intuito de informar, esclarecer e orientar sobre relevantes aspectos da segurança do paciente, demonstrando a igual importância de todos para sustentar a assistência de enfermagem em princípios e fundamentos que promovam a segurança do paciente. Desta forma, acredita-se que a referida cartilha forneça elementos capazes de contribuir para a construção do conhecimento de enfermagem, desenvolvimento profissional e melhora da assistência prestada à população (COREN-SÃO PAULO, 2010).

\subsection{Dimensão cultura de segurança do paciente: "Respostas não punitivas aos erros"}

$\mathrm{Na}$ busca pela qualidade do cuidado à saúde, a garantia da segurança do paciente se constitui um compromisso das instituições e dos profissionais. Desta forma, a redução dos riscos inerentes à prestação da assistência se relaciona diretamente com as mudanças na cultura e nos processos de trabalho adotados pelos serviços de saúde, visto que a assistência produzida e consumida é resultante de um sistema complexo de relações, o que torna passível a ocorrência de erros e/ou eventos adversos no processo do cuidado (Nascimento, 2010).

O problema do erro humano pode ser visto sob duas formas de abordagem, na pessoa e no sistema. A abordagem na pessoa teve um grande impacto no passado e incide sobre atos inseguros devido a processos de esquecimento, desatenção e imprudência, o foco é a culpabilização. A premissa básica na abordagem do sistema é que os seres humanos são falíveis e erros são esperados, mesmo na melhor organização, o foco são as condições em que as pessoas trabalham. Os erros são vistos como consequências, em vez de causas, com origem em fatores sistémicos a montante. Não podemos mudar a condição humana, podemos mudar as condições sob as quais os seres humanos trabalham. A ideia central é a de que o sistema deve ter defesas, e quando um evento adverso ocorre, a questão importante não é saber quem errou, mas sim como e porque as defesas falharam (Reason, 2000).

\section{1o Dimensão cultura de segurança do paciente: "STAFFING"}

O dimensionamento de pessoal de enfermagem é a etapa inicial do processo de provimento de pessoal, que tem por finalidade a previsão da quantidade de trabalhadores por categorias, requeridas para suprir as necessidades de assistência de enfermagem, direta 
ou indiretamente prestada à clientela (Kurcgant, Cunha, Gaidizinski, 1989).

\section{METODOLOGIA}

O presente estudo de intervenção foi realizado em hum hospital do municipio da grande São Paulo, que possui contrato de gestão com a Secretaria de Estado da Saúde desde 2004 é de grande porte com 309 leitos, funciona 24 horas por dia e proporciona atendimento clínico, cirúrgico além de terapia intensiva, que possui o Sistema ONA (Organização Nacional de acreditação) desde o ano de 2006, o hospital busca a excelência do serviço público de saúde. Ações de responsabilidade social e compromisso com os princípios do Sistema Único de Saúde sempre embasaram suas diretrizes. A escolha desse cenário aconteceu por ser um local em que as pesquisadoras trabalham e vivenciam a experiência sobre a cultura de segurança do paciente, com apoio da diretoria de enfermagem foi discutido sobre o objetivo da realização da pesquisa na instituição, não gerando nenhum custo financeiro.

O instrumento validado por REIS (2013) do qual se divide em duas partes: caracterização sociodemográfica e onze dimensões de cultura de segurança do paciente. As perguntas redigidas de forma afirmativas ou negativas obedecendo aos critérios propostos na Escala Likert que trazem uma variação em 4 graus de percepção: "Discordo, Discordo totalmente a Concordo e Concordo totalmente". Quanto à análise das variáveis sociodemográficas; dimensões de cultura de segurança e grau de segurança foram realizadas estatísticas descritivas. Adotou-se como avaliações positivas as que fossem assinaladas como concordo/concordo totalmente ou quase sempre/sempre para as assertivas redigidas de forma afirmativa e as que fossem discordo/discordo totalmente ou raramente/nunca para as assertivas que fossem redigidas de forma negativa. Para fins de análise foram adotados escores de i a 5 , sendo i a pior avaliação e 5 a melhor.

O projeto foi realizado da seguinte forma: disponibilizados computadores na entrada de colaboradores, nas unidades de internação e áreas administrativas, além do encaminhamento do link para preenchimento através de mídia social (WhatsApp), facilitando assim a adesão e a participação das equipes multidisciplinares.

A coleta de dados ocorreu nas dependências do hospital onde foi aplicado um questionário com 42 questões de respostas fechadas, sendo realizado no mês de agosto de 2019, o tempo médio de preenchimento do instrumento foi de dez minutos. 
A população deste estudo foi composta por 772 profissionais atuante na instituição (Médicos, Enfermeiros, Auxiliares e técnicos de enfermagem, Fisioterapeutas, Fonoaudiólogas, Nutricionistas, Psicólogas, Assistentes Sociais, Terapeutas Ocupacionais, Administrativos, Portaria, Hotelaria, dentre outros). Como critérios de exclusão foram considerados os profissionais em licença prolongada, que não estivessem presentes na instituição durante o período da coleta de dados (férias e atestado).

O estudo foi pautado no cumprimento das diretrizes estabelecidas pela Resolução 196/96 do Conselho Nacional de Saúde, do Ministério da Saúde, Comitê de Ética em Pesquisa (CEP) que regulamenta a pesquisa envolvendo seres humanos, a qual incorpora sob a ética do indivíduo e da coletividade a autonomia, não maleficência, beneficência e justiça, visando assegurar os direitos e deveres dos sujeitos da pesquisa (Brasil, 1996).

\section{RESULTADOS}

No resultado da pesquisa realizada foram distribuídos um total de I.ors questionários com um montante de 772 (76\%) de adesão dos participantes que atuam direta ou indiretamente no cuidado prestado ao paciente, o resultado foi divulgado na intranet para todos da equipe multiprofisional. O resultado descrito abaixo ira demonstrar um parcial da pesquisa destacando os pontos frageis que seram trabalhados na isntituição.

Dividimos o resultado em duas etapas que foram as seguintes:

\section{Pontos fortes da segurança do paciente}

$\checkmark$ Itens escritos positivamente que obtiveram $\geq 70 \%$ de respostas positivas ("concordo totalmente" ou "concordo")

$\checkmark$ Itens escritos negativamente que obtiveram $\geq 70 \%$ das respostas negativas ("discordo totalmente" ou "discordo")

\section{Pontos frágeis da segurança do paciente}

$\checkmark$ Itens escritos positivamente que obtiveram $\leq 45 \%$ de respostas positivas ("concordo totalmente" ou "concordo")

$\checkmark$ Itens escritos negativamente que obtiveram $\leq 45 \%$ de respostas negativas ("discordo totalmente" ou "discordo").

As II dimensões avaliadas foram consideradas com Pontos fortes e Pontos frágeis e teve como foco de melhorias internas com curto espaço de tempo, segue abaixo quadro I 
onde demonstram as dimensões que foram avaliadas.

Quadro I - Distribuição das dimensões, sua definição e os resultados obtidos na aplicação da técnica de escala de Likert.

\begin{tabular}{|c|c|c|}
\hline Dimensões & Definição & Áreas \\
\hline $\begin{array}{l}\text { I. Trabalho em equipe no âmbito das } \\
\text { unidades }\end{array}$ & $\begin{array}{l}\text { As unidades do hospital cooperam e se } \\
\text { coordenam entre si para oferecer o melhor } \\
\text { cuidado para o paciente. }\end{array}$ & Fortes \\
\hline $\begin{array}{l}\text { 2. Expectativas e ações de promoção } \\
\text { da segurança dos supervisores e } \\
\text { gerentes. }\end{array}$ & $\begin{array}{l}\text { Supervisor/gerente considera as sugestões da } \\
\text { equipe para melhoria da segurança do paciente, } \\
\text { elogia o trabalhador ou equipe que segue os } \\
\text { procedimentos corretamente e não negligencia } \\
\text { problemas de segurança do paciente. }\end{array}$ & Fortes \\
\hline $\begin{array}{l}\text { 3. Aprendizado Organizacional - } \\
\text { melhoria contínua }\end{array}$ & $\begin{array}{l}\text { Existe uma cultura de aprendizagem na qual os } \\
\text { erros levam as mudanças positivas e as } \\
\text { mudanças são avaliadas em sua eficácia. }\end{array}$ & Fortes \\
\hline $\begin{array}{l}\text { 4. Apoio da gestão hospitalar para a } \\
\text { segurança do paciente }\end{array}$ & $\begin{array}{l}\text { A gestão hospitalar oferece um clima de } \\
\text { trabalho que promove a segurança do paciente e } \\
\text { demonstra que a segurança do paciente é a } \\
\text { prioridade maior. }\end{array}$ & Fortes \\
\hline $\begin{array}{l}\text { 5. Feedback e comunicação a respeito } \\
\text { de erros }\end{array}$ & $\begin{array}{l}\text { Os colaboradores são informados sobre os erros } \\
\text { que acontecem, é dado retorno sobre as } \\
\text { mudanças implantadas e são discutidas } \\
\text { maneiras de prevenir erros com a equipe. }\end{array}$ & Fortes \\
\hline 6. Abertura para as comunicações & $\begin{array}{l}\text { Os colaboradores podem livremente discutir se } \\
\text { observam algo que pode afetar negativamente o } \\
\text { paciente e sentem-se livres em questionar seu } \\
\text { superior. }\end{array}$ & Fortes \\
\hline 7. STAFFING & $\begin{array}{l}\text { Existe número suficiente de colaboradores para } \\
\text { a efetiva execução do trabalho e o número de } \\
\text { horas trabalhadas é apropriado para oferecer o } \\
\text { melhor cuidado ao paciente. }\end{array}$ & Frágeis \\
\hline $\begin{array}{l}\text { 8. Transferências internas e pas- } \\
\text { sagem de plantão }\end{array}$ & $\begin{array}{l}\text { Informações importantes do cuidado do } \\
\text { paciente são transmitidas entre as unidades do } \\
\text { hospital e durante as mudanças de turnos. }\end{array}$ & Frágeis \\
\hline 9. Respostas não punitivas aos erros. & $\begin{array}{l}\text { Os trabalhadores sentem que seus erros e os } \\
\text { eventos reportados não são utilizados contra } \\
\text { eles e que os erros não são anotados em suas } \\
\text { fichas funcionais. }\end{array}$ & Frágeis \\
\hline Io. Percepção geral sobre segurança. & $\begin{array}{l}\text { Os procedimentos e sistemas existentes na } \\
\text { organização são efetivos na prevenção de erros } \\
\text { e não existem problemas quanto à segurança do } \\
\text { paciente. }\end{array}$ & Fortes \\
\hline II. Frequência de eventos relatados & $\begin{array}{l}\text { Com que frequência são notificados os erros nas } \\
\text { diversas modalidades. }\end{array}$ & Fortes \\
\hline
\end{tabular}

Diante dos resultados apresentados foram propostas varias ações de melhoria para os pontos fracos identificados, segue analise e ações propostas: 


\section{STAFFING}

O Gráfico I demonstra que 19\% responderam que tem quantidade suficiente de colaboradores para lidar com a carga de trabalho, realizado levantamento das atividades que os profissionais realizam, dentro de sua unidade de trabalho para alinhamento da assistência prestada ao paciente e distribuição da carga de trabalho, de acordo com os instrumentos disponíveis para avaliação de grau de dependência e instabilidade (FUGULIM, DINI, TISS, NEMS) do doente, conforme área de atuação, 36\% dos colaboradores responderam que trabalha-se a mais do que o necessário para fazer atendimento ao paciente, realizado levantamento do dimensionamento de escala por unidade para trabalhar esta questão, 50\% responderam que utilizam mais colaboradores temporários do que o necessário para fazer o melhor atendimento ao paciente, resultado da rotatividade principalmente da categoria médica devido sermos um hospital escola com residência e por trabalhar com a equipe médica terceirizada, alinhado com os gestores responsáveis para criarem estratégias de retenção de talentos com intuito de não causar impacto na assistência prestada ao paciente, $33 \%$ responderam que trabalhamos em modo de crise, reflexo do alto absenteísmo da instituição e do artigo 394, da CLT que afasta as gestantes e lactantes das atividades insalubres; discutido a questão com os gestores de área para conscientização dos colaboradores quanto aos impactos das ausências, além da liberação da diretoria para contratação de novos colaboradores em substituição aos atualmente afastados.

\section{Gráfico I -- Resultados diante da dimensão Staffing, agosto 2019.}

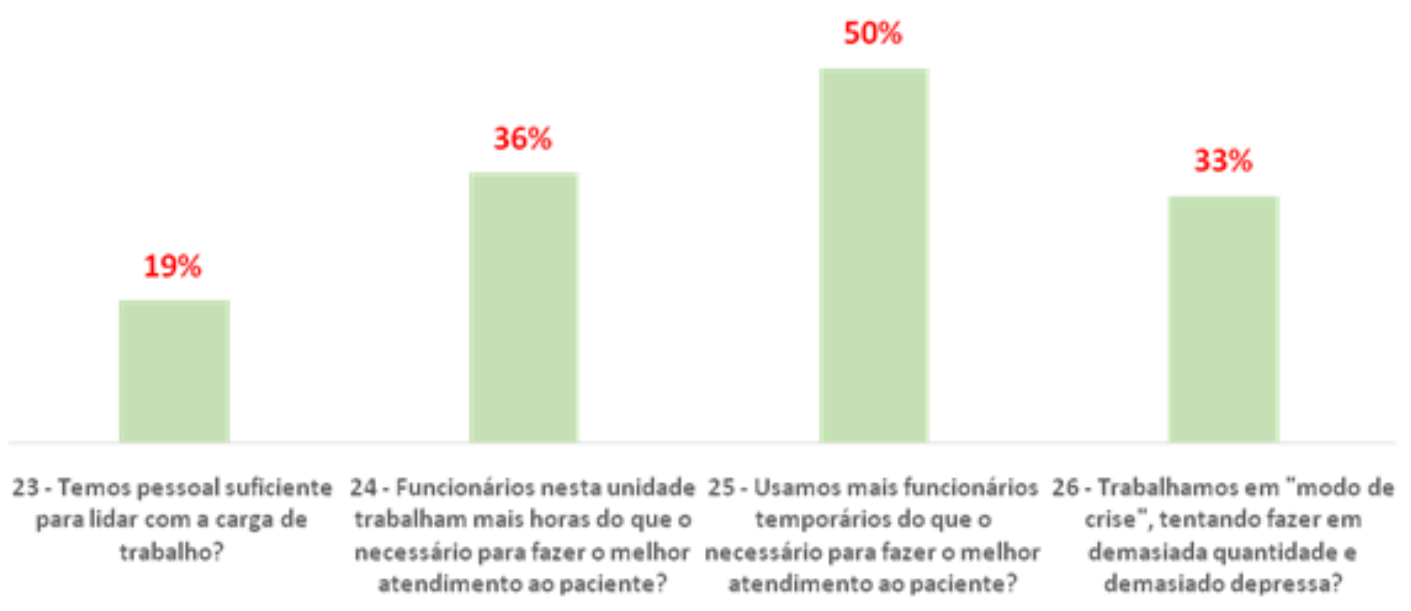

\section{Transferências internas e passagens de plantão}

Equipe de enfermagem é aquela que presta o cuidado durante as 24 horas de internação do paciente, desta forma o trabalho das diferentes equipes devem ser integrados, pois se 
baseiam na continuidade do cuidado. No entanto, para que realmente ocorra a continuidade do cuidado, as equipes devem trocar importantes informações a respeito do tratamento médico e dos cuidados prestados ao paciente (ALMEIDA,20II).

O gráfico 2 demonstra que na percepção dos colaboradores $32 \%$ das informações se perdem na transferência do paciente de uma unidade para outra, adotamos então a utilização impresso de transferência entre unidades que foi reformulado, para melhor eficácia na transmissão de informações entre equipes, $36 \%$ responderam que os problemas frequentemente ocorrem na troca de informação entre unidades do hospital, fortalecido com as equipes que as informação estejam presentes no impresso, que foi reformulado para transmissão das informações (Anexo B e C) e com intuito de atender todas as áreas, 31\% responderam que a prolemática para o paciente está na troca de turno, o método Sbar também foi fortalecido para uma informação clara e efetiva na passagem de plantão, estruturada a equipe de enfermagem para a passagem de plantão, ao Enfermeiro, beira leito alinhando as informações importantes a serem registradas no impresso (filipeta Anexo A), formulado de acordo com as especificidades das áreas para transmissão da informação, 37\% dos colaboradores sentem-se como os seus erros são levantados contra eles, realizado reunião de ro min durante as trocas de turnos para tratativa de possíveis divergências e desvio de protocolos instituidos.

Gráfico 2 - Resultados diante da dimensão Transferências internas e passagem de plantão, agosto 2019.

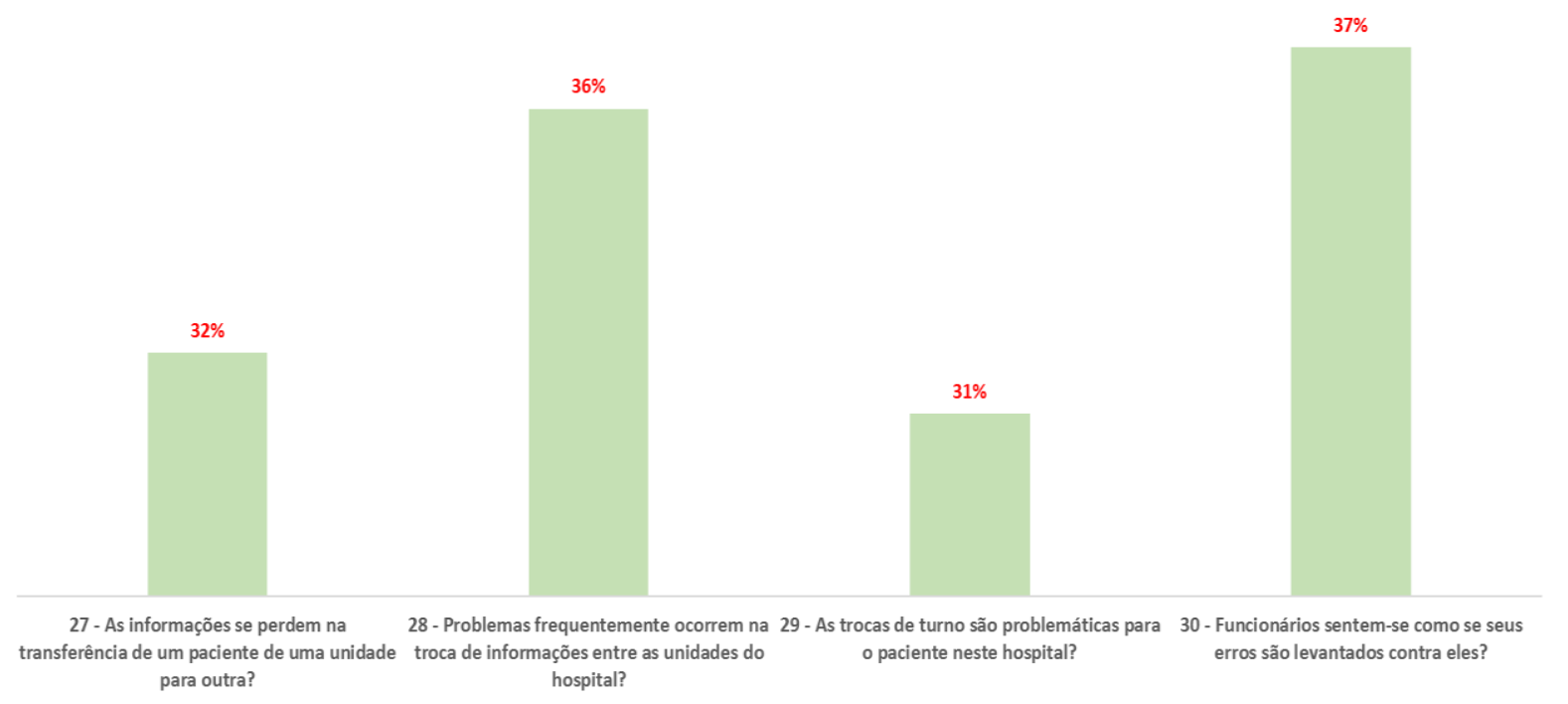




\section{Respostas não punitivas aos erros}

O gráfico 3 demonstra que $31 \%$ dos colaboradores sentem que seus erros são levantados contra eles, os eventos identificados são mensalmente discutidos com todas as equipes de cada período, $29 \%$ responderam que quando um evento é relatado, parece que a pessoa está sendo avaliada e não o problema, discutido o caso juntamente com o núcleo de segurança do paciente, para buscar soluções de melhoria e tratar com o colaborador envolvido a identificação dos motivos reais além de traçar propostas de melhoria, 2.1\% dos colaboradores temem que os erros que cometem sejam mantidos em seu arquivo pessoal, esclarecido aos colaboradores a importância dessa pratica avaliativa e dos motivos que geram registro do ocorrido em prontuário.

Gráfico 3 - Resultados diante da dimensão Respostas não punitivas ao erro, agosto 2019.

$31 \%$

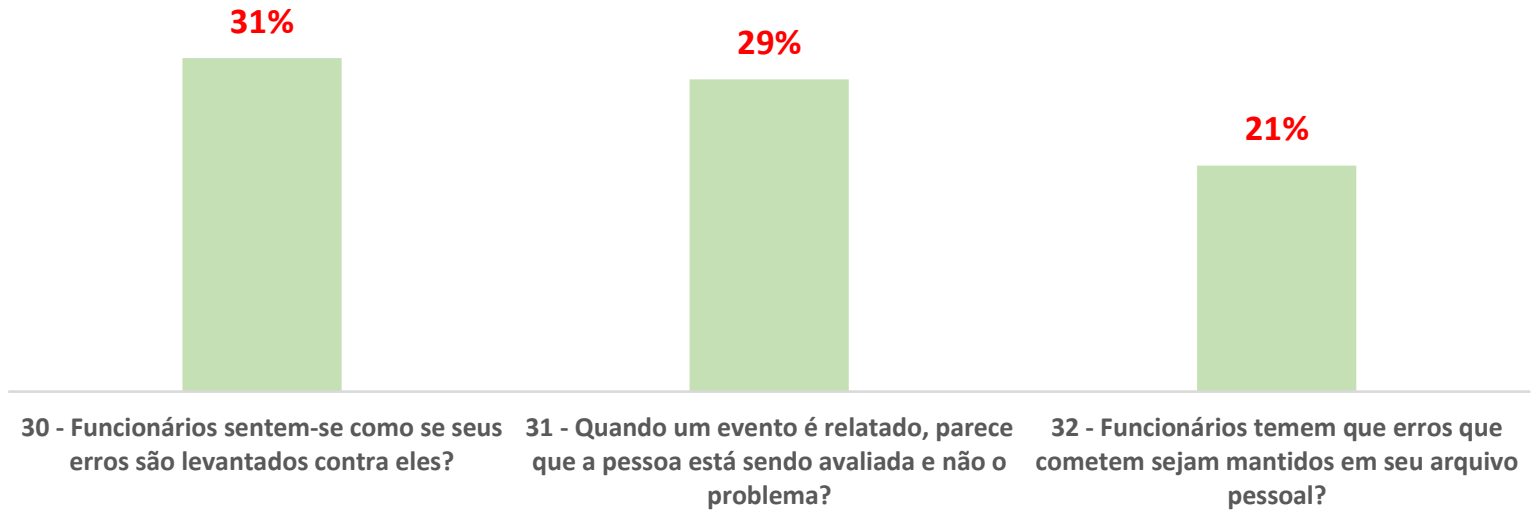

\section{CONSIDERAÇÕES FINAIS}

O presente estudo possibilitou avaliar a percepção dos profissionais de saúde do hospital de ensino da grande São Paulo, através das dimensões de cultura de segurança do paciente bem com identificar os fatores que influenciam diretamente na qualidade e segurança do paciente.

Em relação à percepção do profissional da área da saúde, diante da cultura de segurança do paciente implantada, a pesquisa permitiu concluir que existe compreensão sobre os principais fatores relacionados e seus impactos na qualidade dos cuidados prestados em como na segurança não só do paciente, mas dos colaboradores e da instituição de saúde, organizações com cultura de segurança positiva são aquelas caracterizadas por comunicação 
baseada na confiança mútua, por percepções compartilhadas da importância da segurança e na confiança e eficácia de medidas preventivas.

Por intermédio da análise das percepções dos profissionais quanto às onze dimensões que compõem o questionário conclui-se a existência de pontos positivos na cultura de segurança que serão utilizados para alavancar e mudar o cenário mais frágil existente atualmente, bem como identificar as fragilidades a serem trabalhadas.

Como pontos positivos evidencia-se o trabalho em equipe no âmbito das unidades, a fim de fornecer o melhor cuidado ao paciente e redução da carga de trabalho, quanto a expectativa das ações e promoção da segurança por parte da direção os colaboradores consideram que suas sugestões são aceitas para melhoria dos processos e consequente garantia da segurança, acontecendo elogio ao trabalhador que segue corretamente os procedimentos, a comunicação está aberta quando observam algo que pode afetar negativamente o paciente, o clima de trabalho promove segurança e prioridade maior, quanto ao feedback e comunicação relacionados aos erros, os colaboradores são informados e o retorno relacionado a mudança implantada são discutidos com finalidade de avaliar a efetividade, a frequência dos erros são notificados à equipe nas diversas modalidades e a percepção geral sobre segurança, os procedimentos e sistemas existentes na organização são efetivos na prevenção de erros não existindo problemas quanto a segurança do paciente, os profissionais consideram o apredizado organizacional na qual os erros levam a mudanças positivas e as mudanças são avaliadas em sua eficácia.

Considerando os principais pontos positivos desta pesquisa, podemos destacar a participação de todas as categorias profissionais, envolvimento e aproximação dos gestores, adesão à pesquisa e apoio da diretoria local.

As seguintes fragilidades foram evidenciadas: os profissionais acreditam que a Instituição tem mais funcionários temporários do que precisaria na realização do atendimento, que trabalham mais horas do que o necessário para fazer o melhor para o paciente e que atuam em modo de crise, suas atividades devem ser feitas depressa, sentem se que seus erros são levantados contra eles, e nos casos de eventos acontecidos a avaliação da pessoa acontece ao invés da avaliação do evento e temem que os erros cometidos sejam mantidos em seu prontuário. Observa-se que mesmo os profissionais relatando como problemática a comunicação na passagem de plantão entre as unidades bem como entre os plantões/turnos não tem comprometido o processo de cuidar. 
Um dos pontos a serem trababalhados na imaturaridade da cultura de segurança do paciente refere á comunicação entre as equipes multiprofisionais e seus pares, como ação foi implantado a confecção de uma filipeta que consiste de informações importantes e precisas relacionadas ao paciente, fornecer dados relevantes para tomada de decisão e contiunidade da assitencia bem como informando alergias, procedimentos realizados, utilização de dispositivos invasivos e quaisquer queixas relevantes que surgerem maior atenção ao paciente com o objetivo de evita-las, uma hora antes o Enfermeiro recebe plantão beira leito do Auxiliar de Enfermagem validando as informações, reunião de dez minutos entre as equipes, reformulação do impresso de transferência intra- hospitalar treinamento de passagem de plantão seguindo a ferramenta SBAR aonde irá estruturando a comunicação para reduzir chances de erros e omissões durante a passagem de plantão.

Tais apontamentos se constituem como oportunidades de melhoria e fortalecimento, que permitam direções a seguir quanto à utilização dos recursos existentes ou necessários para que se planeje a assistência à saúde com qualidade, solucionando os problemas existentes, potenciais e contingenciais com melhoria contínua.

Pode-se observar que no resultado desta pesquisa que todas as dimensões apresentadas, são fundamentais para análise e continuidade ao processo de desenvolvimento da cultura de segurança do paciente, para diminuir os impactos dos incidentes das instituições de saúde.

Quanto à percepção geral dos profissionais da saúde referente à cultura da segurança do paciente implantada na instituição ficou nítido o grande interesse em garantir a segurança do paciente por parte de todos os membros da equipe e ainda verificou-se com os resultados que deverá ocorrer maior capacitação profissional, desenvolvendo metas à curto, médio e longo prazos com o objetivo de fortalecer os pontos fortes e mitigar os fracos, levando assim a mudança de cultura na organização para garantirmos a melhoría e progressão contínuas.

\section{REFERÊNCIAS}

I. AUSTRALIA. VICTORIAN DEPARTMENT OF HUMAN SERVICES - Clinical risk management strategy. Victoria: State Government of Victoria, 200I.

2. BARROS CG. Segurança do paciente como prioridade nas organizações hospitalares. [Apresentação do Hospital Albert Einstein; 2013; São Paulo].

3. BATALHA, E.M.S.S.; MELLEIRO, M.M. Cultura de segurança do paciente em um hospital de ensino: diferenças de percepção existentes nos diferentes cenários dessa instituição Texto 
contexto - enferm. v.24 n.2 Florianópolis Apr./June 2015.

4. BERNARDES A, CECILIO LCO, ÉVORA YDM, GABRIEL CS, CARVALHO MB. Modelo de gestão colegiada e descentralizada em hospital público: a ótica da equipe de enfermagem. Rev Latino-Am Enfermagem, v.ı n.4, Ribeirão Preto Jul/Ago. 201 I.

5. BOGNÁR A, BARACH P, JOHNSON JK, DUNCAN RC, BIRNBACH D, WOODS D, et al. Errors and the burden of errors: attitudes, perceptions, and the culture of safety in pediatric cardiac surgical teams. Ann Thorac Surg, v.85, n.4, p.1374-81, 2008.

6. BARBOSA IA, SILVA MJP. Cuidado humanizado de enfermagem: o agir com respeito em um hospital universitário. Rev Bras Enferm. v.6o, n.5, pp.546-551, Brasília, 2007.

7. BRASIL. CONSELHO NACIONAL DE SAÚDE. Resolução n ${ }^{\circ} 196 / 96$ sobre pesquisa envolvendo seres humanos. Bioética. 1996; 4 (2): 15-25.

8. BRASIL. MINISTÉRIO DA SAÚDE; Agência Nacional de Vigilância Sanitária. Resolução RDC n. 36, de 25 de julho de 2013. Institui ações para a segurança do paciente em serviços de saúde e dá outras providências [Internet]. Brasília; 2013.

9. CARVAlHO, R. E. F. L. Adaptação transcultural do Safety Attitudes Questionnaireparapara o Brasil - Questionário de Atitudes de Segurança. 20 II.

Io. CARNEIRO, A. V. - O Erro clinico, os efeitos adversos terapêuticos e a segurança dos doentes: uma análise baseada na evidência científica. Revista Portuguesa de Saúde Pública, v. temático io p.3-ıo. Portugal, 2010.

II. CAON JL. Da existência analfabética à existência alfabetizada. Revista do GEEMPA 6, outubro, p. 37-52. 1998.

12. CAPUCHO, H.C; CASSIANI, S.H. Necessidades de implantar programa nacional de segurança do paciente. Rev. Saúde Pública. v.47, n.4, p.791-798, 2013. Disponível em: . Acesso em: 04 mar. 2017.

13. CAMPOS LF, MELO MRAC. Os desafios da comunicação administrativa na enfermagem. In: 8o Simpósio Brasileiro de Comunicação em Enfermagem; 2002 maio 74-8; Ribeirão Preto, Brasil. Ribeirão Preto: Escola de Enfermagem de Ribeirão Preto da Universidade de São Paulo; 2002.

14. CLARO CM, KROCOCKZ DVC, Toffolleto MC, Padilha KG. Adverse events at the intensive care unit: nurses' perception about the culture of no-punishment. Rev Esc Enferm USP [Internet]. 20II Mar [cited2014 Jul oI]; 45(I):167-72.

I5. CLANTON J, CLARK M, LOGGINS W, HERRON R. Effective handoff 
communication. In: Firstenberg MS, Stawicki S, editors. Vignettes in patient safety. London: IntechOpen; 2018.

16. CONSELHO REGIONAL DE ENFERMAGEM DO ESTADO DE SÃO PAULO. Rede Brasileira de Enfermagem e Segurança do Paciente. Dez passos para a segurança do paciente. São Paulo: Conselho Regional de Enfermagem, zoı.

17. COLLA JB, BRACKEN AC, KINNEY LM, WEEKS WB. Measuring patient safety climate: a review of surveys. Qual Saf Health Care. 2005;14(5):364-6.

18. Dicionário online de Português. Significado de Cultura. Disponível em: <https://www.dicio.com.br/cultura/> Acesso em: I8 jan de 2020.

19. D'INNOCENZO, M.; ADAMI, N.P.; CUNHA, I.C.K.O. O movimento pela qualidade nos serviços de saúde e enfermagem. Rev. bras. Enferm,v.59, n.I, p.84-88, Brasília, 2006.

2o. DONALDSON MS, editor. Measuring the quality of health care. Washington: National Academies Press; 1999.

2I. EAGLETON, T. A idéia de cultura, São Paulo: Editora Unesp, 2003.

22. FLEURY MTL. Cultura e poder nas organizações. $2^{\underline{a}}$ ed. São Paulo: Atlas; 1996.

23. FREITAS M. S. Cultura Organizacional grandes temas em debate. Rev. adm. empres., v.3i n.3, July/Sept. São Paulo, I99ı.

24. . El-Jardali F, Sheikh F, Garcia NA, Jamal D, Abdo A. Patient safety culture in a large teaching hospital in Riyadh: baseline assessment, comparative analysis and opportunities for improvement, 2014.

25. GALT KA, PASCHAL KA. Foundations in patient safety for health professionals. Massachusetts (US): Jones \& Bartlett Learning; 2010.

26. HEALTH AND SAFETY COMISSION. Third Report: Organizing for Safety. ACSNI Study Group of Human Factors. London: HMSO; 1993.

27. HELMAN C.G. Cultura, saúde e doença. $5^{\circ}$ edição. São Paulo: Artmed editora SA,2009. 28. KOHN LT, CORRIGAN JM, DONALDSON MC, editors. Committee on Quality of Health Care; Institute of Medicine. To Err is Human: building a safer health system. Washington (DC): National Academy Press; 2000.

29. KRISTENSEN S, MAINZ J, BARTELS P. PATIENT SAFETY - a Vocabulary for European Application. Aarhus, Denmark: SIMPATIE European Society for Quality in Healthcare - Office for Quality Indicators, 2007.

30. KURCGANT P; CUNHA, K; GAIDIZINSKI, RR. Subsídios para a estimativa de 
pessoal de enfermagem. Enfoque. 1989.

3I. MARQUES, F.L.G.; LIEBER, N.S.L. Estratégias para a segurança do paciente no processo de uso de medicamentos após alta hospitalar. Rev. de Saúde Coletiva, v.24, n.2, p.401-420, Rio de Janeiro, 20I4.

32. MASSAROLO MCKB E FERNANDES MFP. Ética e gerenciamento em enfermagem. In: Kurcgant P. coordenadora. Gerenciamento em enfermagem. $2^{\underline{a}}$ ed. Rio de Janeiro: Guanabara Koogan; 2010.

33. MINISTÉRIO DA SAÚDE (BR). Portaria n. 529 de Io de abril de 2013: Institui o Programa Nacional de Segurança do Paciente (PNSP). Brasília (DF): MS; 2013.).

34. MINUZZ A. P., SALUM N. C., LOCKS M. O. H., Avaliação da cultura de segurança do paciente em terapia intensiva na perspectiva da equipe de saúde. Texto Contexto Enferm, v.25, n.2, Santa Catarina, 2016.

35. NASCIMENTO NB, TRAVASSOS CMR. Medical errors and violation of rules and standards in health: a theoretical discussion in the area of patient safety. Physis. 2010.

36. NIGHTINGALE, F. Notes on Hospitals. 3. ed. London: Longman, Green, Longman, Roberts, and Green, 1863.

37. NIEVA F, SORRA J. Safety culture assessment: a tool for improving patient safety in healthcare organizations. Qual Saf Health Care. 2003 Dec; 12 (suppl III):I7-23.

38. NOGUEIRA J. W. S.; RODRIGUES M. C. S. Comunicação efetiva no trabalho em equipe em saúde: desafio para a segurança do paciente. Cogitare Enfermagem, v.20, n3, p. 636-640, jul/set, Brasília, 2015.

39. OliVEIRA, R.M.; LEITÃO, I.M.T.A.; SILVA, L.M.S.; FIGUEIREDO, S.V.; SAMPAIO, R.L.; GONDIM, M.M. Estratégias para promover segurança do paciente: da identificação dos riscos às práticas baseadas em evidências. Rev. Enf. Esc. Ana Nery. v.ı, n.I, p.122-129, Rio de Janeiro, 2014.

40. PASCHOAL AS, MANTOVANI MF, LACERDA MR. A educação permanente em enfermagem: subsídios para a prática profissional. Rev Gaúcha Enferm, v.27, n.3, Porto Alegre, 2006.

4I. PADILHA KG. Ocorrências iatrogênicas na UTI e o enfoque de qualidade. Rev Latino Am Enfermagem, v.9, n.5, pp.9I-96, Ribeirão Preto, 200I.

42. PANOZZO SJ. Lessons to be learnt: evaluating aspects of patient safety culture and quality improvement within an intensive care unit [thesis]. Adelaide (AU): University of Adelaide; 
2007.

43. PEDREIRA, M.L.G. Enfermagem para a Segurança do Paciente. ACTA Paul. Enferm, v.22, n.4, São Paulo, 2009.

44. PERUZZO, Cicilia M. Krohling. Comunicação comunitária e educação para a cidadania. São Paulo, 2002.

45. PIRES J.C.S., Macedo K. B. Cultura organizacional em organizações públicas no Brasil. RAP, v.40, n.I, p.8I-Io5, Jan./Fev, Rio de Janeiro, 2006.

46. RADUENZ, A. C. et al. Cuidados de enfermagem e segurança do paciente: visualizando a organização, acondicionamento e distribuição de medicamentos com método de pesquisa fotográfica. Rev. Latino-Am. Enferm, v. I8, n. 6, p. 1045-1054, nov./dez, Ribeirão Preto, 2010.

47. REASON J. BEYOND THE ORGANIZATIONAL ACCIDENT: the need for "error wisdom" on the frontline. Qual Safety Heal Care 2004;

48. Reason J. Human error: models and management. BMJ 2000.

49. REIS, C. T. A cultura de segurança do paciente:Validação de um instrumento de mensuração para o contexto hospitalar brasileiro. 2013. 203 f. Tese (Doutorado) - Escola Nacional de Saúde Pública Sérgio Arouca, Fundação Oswaldo Cruz, Rio de Janeiro, 2013.

5o. Rede Brasileira de Enfermagem e Segurança do Paciente (BR). Estratégias para segurança do paciente: Manual para profissionais da saúde. Porto Alegre (RS): EDIPUCRS; 2013.

51. RIGOBELlO MCG, de Carvalho REFL, Cassiani SHB, Galon T, Capucho HC, de Deus NN. Clima de segurança do paciente: percepção dos profissionais de enfermagem. Acta paul. Enferm, v.25, n.5, São Paulo, 2012.

52. SARAIVA L. A. S. Cultura Organizacional em ambiente burocrático. Rev. adm. contemp., v.6, n.I, Jan./Apr., Curitiba, 2002.

53. SCHEIN E. Organizational culture and leadership. San Francisco: Jossey Bass; 1986.

54. SCHEIN E. Three cultures of management: the key to organizational learning. Sloan Manage Rev. 1996;38(I):9-20.

55. SCHNEIDER B, EHRHART MG, MACEY WH. Organizational climate and culture. Annu Rev Psychol. 2013;64: 36r-88.

56. SILVA, M.R.; MATTOS, A.M. Ignaz Semmelweis e a febre puerperal: Algumas razões para a não aceitação de sua hipótese. Filosofia e História da Biologia. v.ıo, n.oı, p.85-98, São 
Paulo, 2015.

57. SORRA JS, NIEVA VF. Hospital Survey on Patient Safety Culture. (Prepared by Westat, under Contract no 290- 96-0004). AHRQ Publication nº 04-004I. Rockville, MD: Agency for Healthcare Research and Quality; Sep 2004.

58. SORRA JS, DYER N. Multilevel psychometric properties of the AHRQ hospital survey on patient safety culture. BMC Health Services Research. 2010.

59. SPEROFF, T. et al. Organizational culture: Variation across hospitals and connection to patient safety climate. Quality and Safety in Health Care, v. 19, n. 6, p. 592- 96, London, 2010.

6o. TASE TH, LOURENÇÃO DCA, BIANCHINI SM, TRONCHIN DMR. Identificação do paciente nas organizações de saúde: uma reflexão emergente. Rev Gaúcha Enferm., v.34, n.3, set, Porto Alegre, 2013.

61. TAVARES, V. H. Segurança do Paciente em Terapia Intensiva: Análise do Uso da Restrição Física. 2013. I29 p. Dissertação (Mestrado) - Departamento de Enfermagem, Faculdade de Ciências da Saúde, Universidade de Brasília, Brasília, 2013.

62. The Canadian Patient Safety Dictionary. 2003[citado Io ami 2011]. Disponível em:〈http://www.biyoetik.org.tr/makaleler/Malpraktis/PatientSafetyDictionary.pdf 〉.

63. OMAZONI A. et al Cultura de segurança do paciente em unidades de terapia intensiva neonatal: Perspectivas da equipe de enfermagem e médica. Rev. Latino-Am. Enfermagem, v.22, n.5, p.755-63, set.-out, Ribeirão Preto, 2014.

64. THOMAS, E. J. [et al] - Incidence and types of adverse events and negligent care in Utah and Colorado. Medical Care. 38:3 (2000) 261-71.

65. THOMAS, E. J.; PETERSEN, L. A. - Measuring Errors and Adverse Events in Healthcare. Journal of General Internal Medicine. I8 (2003) 6I-7.

66. WATCHER RM. Compreendendo a segurança do paciente. Porto Alegre (RS): Artmed; 2010 .

67. World Health Organization $(\mathrm{CH})$. Safe surgery saves lives: the second global patient safety challenge. Geneva: WHO; 2009.

- ZOEHLER KG, LIMA MADS. Opinião dos auxiliares de enfermagem sobre a passagem de plantão. Revista Gaúcha de Enfermagem, v. 2I, no2, Porto Alegre, 2000. 